\title{
Sub-grid modeling of coupled hydrodynamic, vegetative and morphodynamic processes in a salt marsh environment
}

\author{
Mithun Deb, University of Delaware, mdeb@udel.edu \\ James T. Kirby, University of Delaware, kirby@udel.edu \\ Fengyan Shi, University of Delaware, fyshi@udel.edu \\ Guoxiang Wu, Ocean University of China, Qingdao, gxwu@udel.edu \\ Ali Abdolali, National Oceanic and Atmospheric Administration, ali.abdolali@noaa.gov
}

\begin{abstract}
Modeling hydrodynamic processes in a salt marsh system is a challenging task, due to the complexity of topographic features and the scale of such features relative to the overall system size. With growing availability of high-resolution topographic measurements, like LiDAR-derived DEM data, it is increasingly desirable to run a high-resolution model in a large domain and for a long period of time to get trends of sedimentation patterns, morphological change and marsh evolution. However, high spatial resolution poses a big challenge in both computational time and memory storage. Vegetation dynamics in a marsh system needs to be properly taken care of with respect to the vegetation zones and elevation from the mean sea level. The interaction between micro-scale topography and vegetation canopy dominates the overall morphological evolution.
\end{abstract}

Here, we describe a coupled model system for predicting long term hydrodynamics, vegetation biomass, sedimentation and morphological evolution of a salt marsh. The model system couples an existing sub-grid hydrodynamic model (Wu et al., 2016, 2017) with a sediment transport/morphology module and a vegetation biomass module. The sub-grid model of Wu et al. (2016) employed Defina's (2000) approach for integrating finescale information for use in the coarser resolution model used for integration in time. Sub-grid details were treated deterministically, following the approach of Volp et al. (2013). The sub-grid hydrodynamic model performs well in comparison to measured surface elevation and flow data from a relatively small salt marsh system in Delaware Bay, USA (Wu et al 2016, 2017). The computational overhead of determining the sub-grid flow solution in each model grid is fairly light, and the model has been seen to scale relatively well compared to a theoretical factor of (grid resolution) ${ }^{5 / 2}$, with the sub-grid model showing minimal loss of accuracy relative to a full model run at the sub-grid resolution. The sediment transport model employs the sub-grid bedload transport equation of Volp et al. (2016). Vegetation biomass is predicted by a simple logistic equation model proposed by D'Alpaos et al. (2007). The biomass component is loosely coupled with hydrodynamic and sedimentation models owing to the different time scales of the physical and ecological processes. The model is being applied to Delaware marsh evolution in response to rising sea level and changing sediment supplies.

Marsh platform features such as rills and cuts through channel berms contribute to the control of flow patterns and the draining and filling of the platform. These small features are often missing in the model grid due to both incomplete resolution of the features in data sources such as LiDAR as well as loss of resolution in the development of DEM's and model grids. Determination of the size and frequency of occurrence of such features in a general setting is challenging, as many of these small scale topographic features may be hidden under highdensity vegetation canopies and are thus not easily recognized using even higher resolution techniques such as Structure from Motion (SfM). Loss of resolution and representation of these features can lead to modeling defects such as artificial ponding and resulting inaccuracies in important processes such as sedimentation rates and species selection and growth rates of vegetation canopy.

The use of sub-grid approaches in modeling can provide the needed computational efficiency to resolve such features in model grids, but a deterministic representation of the structures may not be feasible to obtain. Recently, we have introduced a stochastic porosity parameter in the model in order to improve the wetting and drying phenomena in artificially isolated depressions. The approach follows the original suggestions of Defina (2000), but we employ a different set of assumptions about the vertical distribution of topographic irregularities, which here are chosen to represent mainly the marsh platform defects. The additional stochastic porosity has been implemented in the sub-grid model system. The paper will discuss verification of the approach in comparison to an extensive set of measurements obtained in the Bombay Hook National Wildlife Refuge, Delaware USA.

\section{REFERENCES}

D'Alpaos, A., Lanzoni, S., Marani, M. and Rinaldo, A. (2007): Landscape evolution in tidal embayments: Modeling the interplay of erosion sedimentation and vegetation dynamics, J. Geophys. Res., 112, F01008.

Defina, A. (2000): Two-dimensional shallow flow equations for partially dry areas. Water Resources Research, 36(11), 3251.

Volp, N. D., Van Prooijen, B. C. and Stelling, G. S. (2013): A finite volume approach for shallow water flow accounting for high-resolution bathymetry and roughness data. Water Resources Research, 49(7), 4126-4135.

Volp, N. D., van Prooijen, B. C., Pietrzak, J. D. and Stelling, G. S. (2016): A subgrid based approach for morphodynamic modelling. Advances in Water Resources, 93, 105-117.

Wu, G., Shi, F., Kirby, J. T., Mieras, R., Liang, B., Li, H. and Shi, J. (2016): A pre-storage, subgrid model for simulating flooding and draining processes in salt marshes. Coastal Engineering, 108, 65-78.

Wu, G., Li, H., Liang, B., Shi, F., Kirby, J. T. and Mieras, R. (2017): Subgrid modeling of salt marsh hydrodynamics with effects of vegetation and vegetation zonation. Earth Surface Processes and Landforms, published online doi:10.1002/esp.4121. 